\title{
Using a New Routing Metric in Paths Discovery Process for MAODV
}

\author{
Read Jabri, Saher Manaseer, Esra'a Mahmoud Alhenawi \\ Department of Computer Science, King Abdullah II School of Information Technology, The University of Jordan, \\ Amman, Jordan \\ Email: jabri@ju.edu.jo, saher@ju.edu.jo,esra_a_2008@live.com
}

How to cite this paper: Jabri, R., Manaseer, S. and Alhenawi, E.M. (2018) Using a New Routing Metric in Paths Discovery Process for MAODV. Open Access Library Journal, 5: e4425. https://doi.org/10.4236/oalib.1104425

Received: February 18, 2018

Accepted: April 15, 2018

Published: April 18, 2018

Copyright () 2018 by authors and Open Access Library Inc.

This work is licensed under the Creative Commons Attribution International License (CC BY 4.0).

http://creativecommons.org/licenses/by/4.0/

(c) (i) Open Access

\begin{abstract}
The limited lifetime period of wireless nodes of Ad hoc networks due to their battery power, has been forming a significant issue and concern for researchers. To solve this previous related research has ignored low energy nodes regardless of its total residual energy without ignoring the importance of hop count value for selecting paths in forwarding decision. This paper aims at finding and proposing a new path selection metric that takes into account the minimum residual energy of all route nodes and hop count value by using the ratio between them for arranging paths in MAODV routing protocol where the discovered paths have been checked periodically for ensuring their availability at any time using special packets, called DTC. The experiment used the GloMoSim simulator to assess the performance of the proposed protocol by comparing it with other protocol that arranging paths using the sum of their nodes remaining energy based on packet delivery ratio, end to end delay and network lifetime. Simulation results show that the proposed routing protocol improves packet delivery ratio by $9.4 \%$ and network lifetime by an average of $21.7 \%$. Also, it provides $14.6 \%$ lesser delay.
\end{abstract}

\section{Subject Areas}

Applications of Communication Systems, Communication Protocols, Green Networking, Network Modeling and Simulation

\section{Keywords}

MANETs, Ad-Hoc Networks Lifetime, Minimal Nodal Residual Energy, DTC_Packets, Glomosim

\section{Introduction}

A mobile ad hoc network (MANET) is an infrastructure-less type of networks. 
No infrastructure is needed to carry out the operations in the network. Nodes in the MANET topology are independent and able to move freely, more over this type of networks doesn't need routers; hence each node is responsible for forwarding packets and exchanging them all along the packet traffic route [1].

Routing protocols are classified into two main protocols according to how information is obtained. First, proactive or table-driven routing protocols such as Distance Vector routing protocol (DSDV) in which all nodes generally store their information in routing tables, which are updated if any change in the network topology happens. In proactive routing protocols, it costs more than the second type named the reactive protocol (on demand), resembling the Ad-Hoc On-demand Distance Vector (AODV) routing protocol which works on demand to discover the needed route. Although this reduces the control message overhead, the delay increased due to route discovery process, yet; the delay can be reduced by using multipath reactive routing protocols [2].

Energy consumption is a significant concept in ad hoc networks since mobile nodes have a limited energy in their batteries. So, minimizing the energy consumption of nodes is the most critical issue to increase the lifetime of ad hoc networks because power failure of a mobile node affects the node itself and decreases network performance due to link failure.

This paper aims at considering the ratio between residual energy of the node and hope counts as a new metric to choose the route path in MAODV, which periodically check the paths to ensure their availability at any time using special packets called DTC.

\section{Related Works}

Developing energy aware routing protocols has become a significant issue in the networks field research. Many studies have taken into consideration the power consumption issue and tried to manage the energy through proposing and conducting many experiments, and modifications were suggested to the single path selecting AODV protocol, to solve the problem of the energy consumption. The remaining energy of the node and number of packets buffered were taken in into account, to select the route between the source and destination in [3]. In another experiment the remaining energy for each intermediate route between source and destination was calculated, and select the route according to its nodes residual energy, by comparing the calculated energy and comparing it to the minimum threshold required value as discussed in [4].

An energy saving protocol was proposed, in which selecting the routes was made according to the residual energy of neighboring nodes. If the measured value was less than the minimum threshold, the node goes into sleep mode. Otherwise, it is selected to be part of the desired route [5]. Shukla et al. proposed a new energy efficient algorithm in [6] that concentrates on finding out the best route between source and destination based on two factors: distance between nodes and battery status of every node in the network. 
One major disadvantage of the single path AODV is that it consumes a lot of time and causes a high overhead due to the process of selecting the best path only and discards any other options. So, every failure cases the discovery process and search for an active route is repeated. This led to the importance of having a multi routes (Multipath) AODV known as MAODV routing protocol, in which the single path algorithm is modified to find multiple disjoints paths from source to destination nodes. Thus, if a failure happens the alternative paths are used to reduce time and overhead caused by repeating the discovery processes. A new protocol was proposed by Cho and Aungin [7], using MAODV algorithm and named "EE-AODV" which arranges the discovered path according to the sum of all nodes residual energy in every path.

The problem with this method is that the total summation of the energy in a path may exceeds the other paths which give it the highest priority, yet some nodes in this path may be dead on the contrary some low energy paths may have the least total yet every node is active, which makes using the minimum remaining energy of a route nodes better than using the total sum.

The other problem appeared is that the multiple paths when selected, are listed so they are used, but their availability checking process is not repeated when they are needed. This can cause the risk of using a route that is not available after a while even if it was listed and stored due to the high mobility of the MANETs.

One of this paper purposes was to solve this problem; by making the source node periodically sends a special packet, called detected (DTC), for ensuring that the alternate paths which discovered in path discovery process and stored at source node remains available; the packet is sent to the destination along each of its alternate path for checking validity of these paths for maintaining in primary path failure time. DTC packets similar in idea not in purpose with Hello packets, special Echo packets that the receiver just timestamps and sends it back in adaptive routing protocols which change their routing decisions to reflect changes in the topology as well as traffic in networks such as distance vector routing protocols as illustrated in [8] and request to send (RTS), clear to send (CTS) packets that have been used in wireless LAN that uses multiple access with collision avoidance protocols, as proposed in [9].

Figure 1 illustrates a scenario where EE-AODV routing protocol which depends on the total sum of the nodes energy, the figure shows two paths between the source node (S) and the destination node (D), as shown sum of residual energy for all nodes in path $1(\mathrm{~S}, 1,3,5, \mathrm{D})$ is greater than it in path $2(\mathrm{~S}, 2,4, \mathrm{D})$, so based on what is discussed in [7] path 1 will be selected for transmitting data via it but this selection will not be suitable here because path 1 contains node- 5 with $\mathrm{RE}=10$ which will be dead before all nodes (node- 2 and node-4) in path 2 . However, Figure 2 provides a scenario illustrated the importance of hop count value in routing decision.

In another experiment, an improved label propagation algorithm was introduced to reveal the overlapping community structure. The algorithm works by 


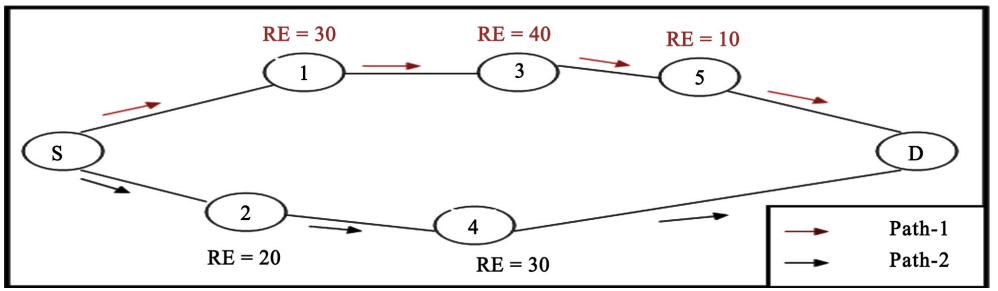

Figure1. Example of the first scenario.

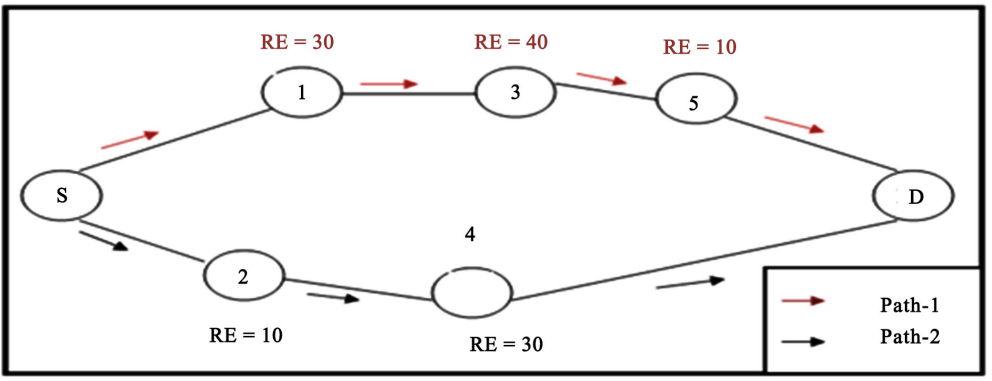

Figure 2. Example for the second scenario.

calculating the variance of each node and the proposed threshold as well as calculating the average energy of the nodes. If the variance is less than the threshold the node is labeled as a bridge node. Using different values for the threshold can reveal more bridge nodes each time. The algorithm proven that it could uncover the overlapping in the termination of the iteration cases as introduced in [10], so the energy of nodes can be used to control many issues in the networks.

For solving the problem of unfair distribution of energy on the nodes of the network, a Node power MAC protocol was proposed and an adaptive listening time for wireless sensor nodes was presented in [11]. The algorithm outperformed the performance comparing to Sensor MAC and Layer MAC, and showed an enhancement of energy consumption by saving $61.1 \%$ of the energy that SMAC and LMAC consumes.

A hierarchal approach was proposed and used by [12], the approach is constructed of many phases, discover, clustering and aggregation. The aggregation is done by grouping the nodes of the same or nearly the same levels of energy. The topology is periodically restructured to assure fair energy distribution among nodes. This method helped in generating energy maps with less costs and energy consumption.

The literature shows the importance of the energy of the nodes and the power consumption of the networks and how it can affect performance and throughput of the network.

\section{The Proposed Routing Protocol}

In this work some modifications have been done on the traditional multi path ad-hoc on-demand distance vector (MAODV) routing protocol-which arranged the discovered paths based on a number of hops-by using a minimum remaining energy of all route nodes and hop count value into account for storing 
the discovered paths in descending order in the source node routing table, in subsection 3.1 below the required modifications illustrated, however protocol processes shown in 3.2 and work phases discussed in

\subsection{Required Modifications on the Structure of AODV Routing Protocol}

Modification on the structure of MAODV routing table: by adding new field in the structure of the traditional MAODV routing table for storing needed information about the route residual energy.

Modification on RREQ control packets: by adding new field in request packet header called residual energy which updates at each intermediate node that receive the request packet for storing the minimum residual energy exists among all nodes through the route from source to destination. At destination node rout residual energy that exists in RREQ header must be stored as a route residual energy.

Modification on RREP control packets: replay packet must be modified in the same way as have been done in request packet to store the residual energy of the reversed route which will be stored in the source node routing table and used for sorting multiple paths that discovered from this source to destination pairs.

\subsection{The Proposed Routing Protocol Processes}

Like traditional MAODV the proposed routing protocol has two processes with some modifications as illustrated here:

\section{1) Route discovery process}

Route discovery process begins when the source node broadcast request packets to all of its neighbors where in each packet there is another field representing the residual energy of the route which initially equal to the remaining energy of first node over the route. Each intermediate node receives the RREQ control packet calculates its residual energy using eq1 that take into account the node past activity and spends simulation time and compares its remaining energy with the energy value which stored in the receiving RREQ packet header, if its own energy is lesser then it updates the residual energy field in that request packet header to save the minimum residual energy in it. This process progresses until the first request packet reach the destination which generates replay packet that updated in the same way. At source node the discovered paths stored in descending order in the routing table based on the ratio of their remaining energy and hop count value called "Ratio".

This simulation has been carried out on a computer with the following specs:

a) Core 2 quad processer with $2.4 \mathrm{GHz}$ processing speed

b) 6 GB of RAM

c) $8 \mathrm{MB}$ of cache memory

\section{2) Route maintenance process}

In route maintenance process the source node chooses the route with highest 
"Ratio" value to transmit data over it. If the selected path fails, many activities must be done, first of all a route error (RERR) control packet will be generated to notify any node using this route to mark it as deactivated route in their routing tables. If this route still needed to complete transmission of the remaining data then transmission turned to an available alternative path that has the maximum "Ratio" value among all of the rest paths that are discovered in discovery process and stored in routing table in descending order. To ensure that the alternate paths stored at a source node still available; the source node must periodically sends a special packet, called detected (DTC), to the destination along each of its alternate paths where the destination just receives it and sends it back. At each time if the detected packet doesn't return again to the source node using any alternate path, this path must be deleted because it is unavailable.

\subsection{Work Phases}

This paper work divided into two phases to accomplish it.

- Phase One: Finding minimum residual energy of all route nodes for each discovered path between source \& destination.

- Phase Two: Arranging multi route in descending order based on energy and number of hops.

\subsection{Description of Project Phases}

\section{Phase-1 $\rightarrow$ Finding minimum residual energy of all route nodes for each discovered path}

Several changes are needed in MAODV route discovery procedure that had been implemented in phase_1 for computing the minimal nodal residual energy of each route between source-destination pairs. So routing table must have new field called Path Power. Each RREQ and RREP must have an additional field called Power which represents the current node power and calculated from Equation 1 and Equation 2 below.

Node Power $(\mathrm{NP})=$ InitPower $-\left(\left(\right.\right.$ Sim_Time ${ }^{*}$

TimeFactorPowerConsumption) + (Activity *

ActivityFactorPowerConsumption)).

Equation 1. NodePower (NP).

Activity = numRequestSent + numReplySent+numRerrSent

+numRerrResent + numDataSent + numDataTrans +

numDataReceived.

Equation 2. Activity of the node.

- Calculating Residual Power Equation

The residual energy had been calculated in each node in any path from source to destination based on the following equation (Equation 1): 


\section{Where:}

- InitPower: initial power that represents the initial node power which I had been assumed random value.

- Sim_Time: simulation time.

- TimeFactorPowerConsumption: constant that represents the percentage of power that will be consumed from the node initial power in the idle state that depends on the temperature.

- ActivityFactorPowerConsumption: constant that takes random value to represent the percentage of power that will be consumed from the node initial power due to activities accomplished by this node.

Activity: activities done by node in the network that depends on seven parameters as illustrated in Equation 2.

Where:

- NumRequestSent: it is the number of RREQ (request) packets that had been transmitted.

- NumReplySent: it is the number of RREP (replay) packets that had been transmitted.

- NumRerrSent: it is the number of RERR (errors) packets that had been transmitted.

- NumRerrResent: it is the number of RERR (errors) packets that had been resent.

- NumDataSent: it is number of data packets that had been sent.

- NumDataReceived: it is number of data packets that had been received.

Based on the previous equations we find the remaining energy in each node in the route and take the minimal node energy to represent the energy of the path.

Phase-2 $\rightarrow$ Arranging multi routes in descending order based on energy and number of hops:

The discovered routes had been arranged based on the ratio between the minimum residual energy of all route nodes and number of hops in descending order. If there exist two or more routs have the same ratio the arrangement must be done randomly.

\section{Simulation Setup and Evaluation Metrics}

This section in this paper illustrates the GloMoSim simulator setup that had been used in experiments for comparing the performance of the proposed routing protocol and protocol proposed in [7] based on end to end delay, packet delivery ratio and network lifetime.

\section{Experiment \# 1:}

The proposed protocol had been applied on random network topology that has the configuration presented in Table 2 to compare its performance with the performance of EEMAODV routing protocol in [7] based on packet delivery ratio for different number of nodes (Table 1).

Experiment \#2: 
Table 1. Parameters of experiment 1.

\begin{tabular}{cc}
\hline Parameter & Value \\
\hline Simulation Time & $1000 \mathrm{sec}$ \\
Simulation Area & $1500 \mathrm{~m} \times 1500 \mathrm{~m}$ \\
Number of Nodes & $4,8,20,50,150$ \\
Routing Protocols & Proposed MAODV, EEMAODV \\
\hline
\end{tabular}

Table 2. Parameters of experiment 2.

\begin{tabular}{cc}
\hline Parameter & Value \\
\hline Simulation Time & $1000 \mathrm{sec}$ \\
Simulation Area & $1500 \mathrm{~m} \times 1500 \mathrm{~m}$ \\
Number of Nodes & $50,100,150,200,250,300$ \\
Routing Protocols & Proposed MAODV, EEMAODV \\
\hline
\end{tabular}

The network configuration that illustrated in below had been used for comparing the proposed protocol with EEMAODV routing protocol in [5] based on end to end delay criterion using different scenarios with various numbers of nodes.

\section{Experiment \#3:}

The effect of the proposed protocol had been tested the network lifetime over different number of nodes using network configuration that had been presented in Table 3.

\section{Results and Discussion}

Figure 3 illustrates the result of five scenarios for measuring packet delivery ratio with varying number of nodes (network density). It shows that packets delivery ratio for the two routing protocols decreases in dense network (number of nodes large; ex: 150_nodes) due to packet collisions the retransmissions of packets increased which decreasing the available bandwidth for data transmission so number of delivered packets decreased. In sparse network (number of nodes small; ex: 4, 8_nodes) because in sparse networks there is a limited number of routes between source and destination, which leads to jam which block packets from reaching the destination. But, the proposed protocol in this paper improves the packet delivery ratio in the network.

Figure 3 presents the impact of number of nodes in the network on the performance of the two routing protocols in terms of end-to-end delay. It shows that the end-to-end delay for each of the routing protocols increases at all scenarios. This is due to the fact that in dense network more number of routing packets is generated and transmitted and hence the interference between neighbor nodes, packet collisions and channel contention increases. Therefore, the time required to reach to destination increases. On the other hand when the network is sparse, due to poor connectivity the routing packets fail to reach to destination 
nodes and thus increase the end to end latency. In Figure 4, we observe that an end to end delay of the proposed routing protocol is smaller than it in EEMAODV proposed in [7]. The reason is that EEMAODV does not consider hop count as a cost metric. As a result, a path which has more hops could be selected for data transmission during route discovery which results an increase in end to end delay.

Figure 5 shows how network lifetime varies by varying density over a network. It is clear that our proposed routing protocol MEAODV satisfies better network lifetime values than original MAODV because it uses paths based on their residual energy.

Table 3. Parameters of experiment 3.

\begin{tabular}{cc}
\hline Parameter & Value \\
\hline Simulation Time & $1000 \mathrm{sec}$ \\
Simulation Area & $1500 \mathrm{~m} \times 1500 \mathrm{~m}$ \\
Number of Nodes & $8,15,20,50,120$ \\
Routing Protocols & Proposed MAODV, EEMAODV \\
\hline
\end{tabular}

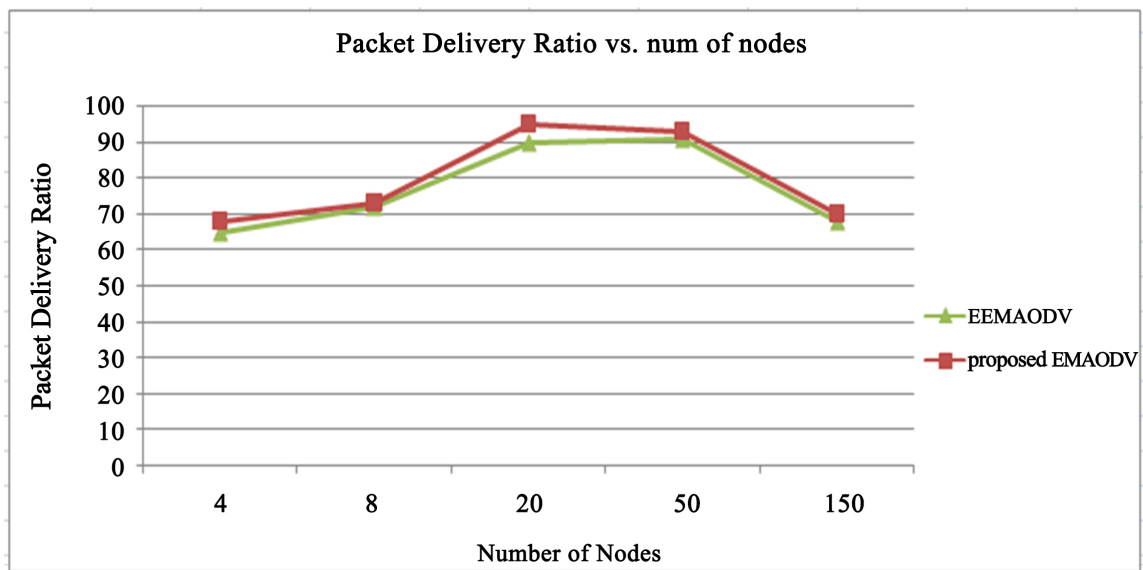

Figure 3. Packet delivery ratio VS. num of nodes.

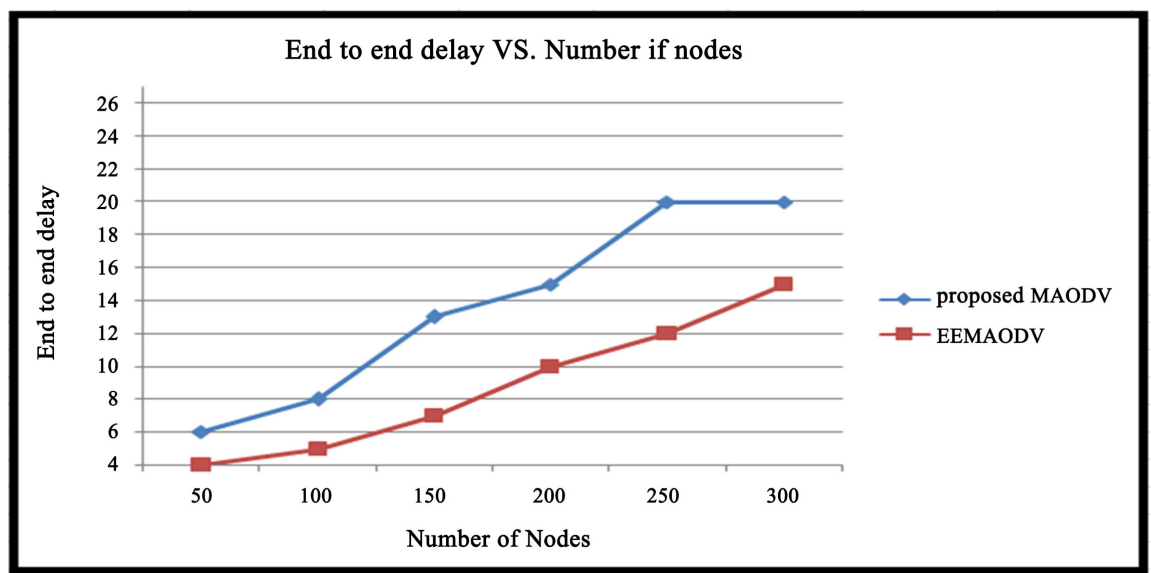

Figure 4. End to end delay VS. num of nodes. 


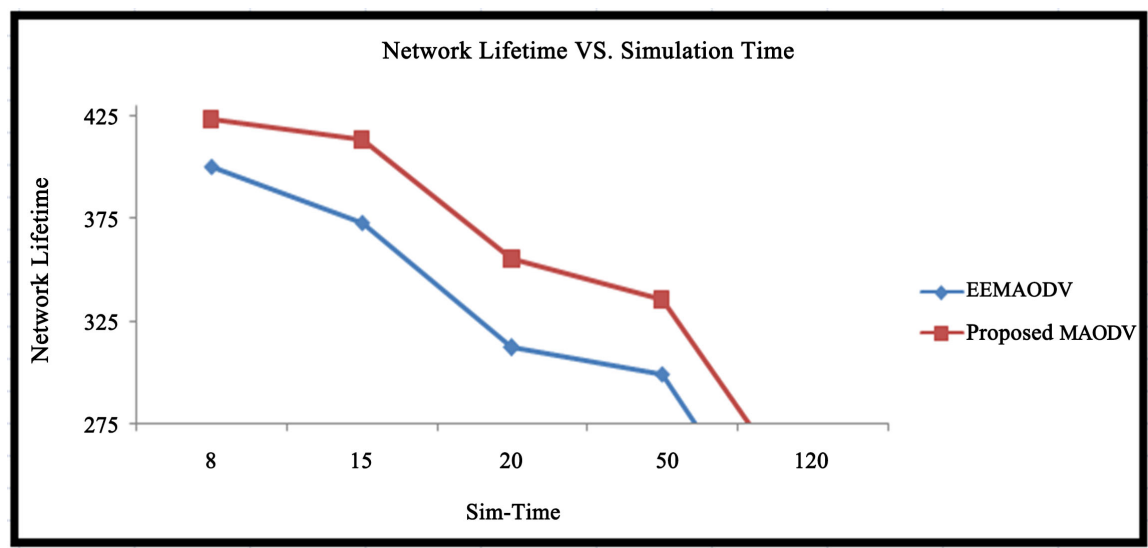

Figure 5. Network lifetime VS. simulation time.

\section{Conclusion}

In this paper a modification on the traditional MAODV routing protocol had been proposed by using the ratio of minimum residual energy in route and hop count value for ordering an on-demand multiple disjoint paths which periodically monitoring for checking their availability. It is able to eliminate failing routes and thereby reduce the number of data packets dropped due to the use of these invalid paths. The performance of the proposed routing protocol had been compared with EEMAODV that ordering paths depending on the sum of their nodes remaining energy using Glomosim simulator based on end to end delay, packet delivery ratio and network lifetime as evaluation criteria over different numbers of nodes. Simulation results show that the proposed routing protocol outperformed on EEMAODV as it improves the packet delivery ratio, network lifetime and has lesser delay than EEMAODV routing protocol.

\section{References}

[1] Manaseer, S., Alsoudi, D. and Aljawawdeh, A. (2017). Border Node Detection: A New Experimental Approach. Journal of Computer Modelling \& New Technologies, 21, 37-40.

[2] Bhatia, D. and Sharma, D.P. (2016) A Comparative Analysis of Proactive and Reactive Routing Protocols over Open Source Network Simulator in Mobile Ad Hoc Network. International Journal of Applied Engineering Research, 11, 3885-3896.

[3] Verma, S., Agarwal, R. and Nayak, P. (2012) An Optimized Energy Aware Routing (OEAR) Scheme for Mobile Ad Hoc Networks Using Variable Transmission Range. International Journal of Computer Applications (0975-8887), 45, No. 12.

[4] Maheshbhai, L.A. and Wandra, K.H. (2014) Adhoc On-Demand Distance Vector-Energy Efficient Approach. International Journal of Computer Applications, 102, No. 8.

[5] Nema, T., Waoo, A., Patheja, P.S. and Sharma, S. (2014) Energy Based AODV Routing Algorithm with Sleep Mode in MANETs. International Journal of Computer Applications, 58, No. 19.

[6] Shukla, R.N. and Shukla, R.K. (2013) Improved Energy Efficiency in AODV. International Journal of Engineering Research \& Technology(IJERT), 2, No. 12. 
[7] Cho, M. and Aung, M. (2014) Energy Efficient Multipath Routing for Mobile Ad Hoc Networks. International Journal of Information Technology, Modeling and Computing (IJITMC), 2, No. 3.

[8] Beniwal, S. and Kumar, D. (2014) An Evolutionary Approach to Distance Vector Routing. International Journal of Latest Research in Science and Technology, 3, 201-205.

[9] Cambazoglu, S. and Sari, A. (2015) Collision Avoidance in Mobile Wireless Ad-Hoc Networks with Enhanced MACAW Protocol Suite. International Journal of Communications, Network and System Sciences, 8, 533. https://doi.org/10.4236/ijcns.2015.813048

[10] Peng, H., Zhao, D., Li, L., Lu, J., Han, J. and Wu, S. (2016) An Improved Label Propagation Algorithm Using Average Node Energy in Complex Networks. Journal of Physica A, 460, 98-104. https://doi.org/10.1016/j.physa.2016.04.042

[11] Ramadan, K., Dessouky, M., Abd-Elnaby, M. and Abd El-Samie F., (2018) Node-Power-Based MAC Protocol with Adaptive Listening Period for Wireless Sensor Networks. International Journal of Electronic Communications, 84, 46-56. https://doi.org/10.1016/j.aeue.2017.10.034

[12] Chan, E. and Han, S. (2009) Energy Efficient Residual Energy Monitoring in Wireless Sensor Networks. International Journal of Distributed Sensor Networks, 5, 1-23. https://doi.org/10.1080/15501320902876055 